\title{
Editorial
}

\section{The Psychotic Depression Assessment Scale (PDAS) in measurement-based care of patients with psychotic depression}

The Hamilton Depression Scale (HAM-D) (1) and the Brief Psychiatric Rating Scale (BPRS) (2) were developed to measure the changes in clinical states resulting from treatment with antidepressant and antipsychotic drugs, respectively. In measurementbased care of depression or schizophrenia the HAM$\mathrm{D}$ and the BPRS have been considered as item banks from which short and valid subscales can be derived to increase the responsiveness, for instance, in demonstrating dose-response relationship of antidepressants or antipsychotics (3).

The Psychotic Depression Assessment Scale (PDAS) has been developed and validated by Østergaard et al. $(4,5)$ in using the most relevant items from the HAM-D and BPRS banks. Thus, the PDAS contains the six items in the HAM-D ${ }_{6}$ subscale (6): depression mood, guilt, work and interests, psychomotor retardation, psychic anxiety, and somatic symptoms (tiredness and pains). From BPRS a five-item subscale has been included: hallucinatory behaviour, unusual thought content, suspiciousness, emotional withdrawal, and bunted affect.

In this short communication, Köse and Østergaard (7) have evaluated the PDAS when rested in itself by a semi-structured interview. This evaluation has covered the two clinimetric analyses as described by Bech et al. (8), namely both a microanalysis and a macroanalysis. In the microanalysis it is evaluated to what extent the items in the scale fulfil the test of scalability (e.g. the coefficient of homogeneity) for using the total score as a sufficient measure of symptoms severity on the dimension being examined. The macroanalysis is concerned with the standardisation of the total score, for example, when identifying the cut-off score for remission in patients treated for psychotic depression. Köse and Østergaard (7) have demonstrated an acceptable scalability of the PDAS with a coefficient of homogeneity above the level of 0.40 , in contrast to the full HAM-D ${ }_{17}$ or BPRS $_{18}$. Using a cut-off score of less than 8 on PDAS Köse and Østergaard (7) obtained a remission percentage of $74 \%$ at endpoint in their 6 weeks trial including both unipolar and bipolar depressed patients. As most of these psychotic depressed patients have received electroconvulsive therapy (ECT), a remission rate of $74 \%$ is in accordance with the classical Medical Research Council (9). In the other classical ECT study, The Northwick Park Electroconvulsive Therapy Trial (10) the superiority of real ECT over simulated ECT in a 4-week treatment period was found in severely depressed patients fulfilling the Newcastle diagnostic scale (11), that is, psychotic depression. The mean HAM- $\mathrm{D}_{17}$ score was in this study at baseline approximately 30 but from this information the degree of psychotic depression is not clear. A score on the PDAS would have been very informative.

The Newcastle depression scale is a diagnostic rating scale to predict response to ECT, not a scale measuring the change in the clinical state resulting from treatment with ECT. To the best of my knowledge, we have no other scales than the PDAS for measuring outcomes of treatment for psychotic depression. In measurement-based care of patients with psychotic depression when ECT often is the choice of treatment, the PDAS should be included.

\section{Per Bech $\dagger$ \\ Psychiatric Research Unit \\ Psychiatric Centre North Zealand \\ Hillerød, Denmark \\ $\dagger$ Deceased May 9th 2018}

\section{References}

1. Hamilton M. Development of a rating scale for primary depressive illness. Br J Soc Clin Psychol 1967;6: 278-296.

2. Overall JE, Gorham DR. The brief psychiatric rating scale. Psychol Rep 1962;10: 799-812.

3. ВеCH P. Measurement-Based Care in Mental Disorders. New York: Springer, 2016.

4. Østergaard SD, Pedersen CH, Uggerby $\mathrm{P}$ et al. Clinical and psychometric validation of the Psychotic Depression Assessment Scale. J Affect Disord 2015;173: 261-268. 


\section{Bech}

5. Vermeulen T, Lemey L, Van Diermen L et al. Clinical validation of the Psychotic Depression Assessment Scale (PDAS) against independent global severity ratings in older adults. Acta Neuropsychiatr 2018;1-6. ePub ahead of print: https://doi.org/10.1017/neu.2018.2.

6. Bech P, Allerup P, Gram LF et al. The Hamilton Depression Scale. Evaluation of objectivity using logistic models. Acta Psychiatr Scand 1981;63:290-299.

7. Köse C, ØsteRGAARD SD. Validation of the semi-structured Psychotic Depression Assessment Scale (PDAS) interview. Acta Neuropsychiatri 2018;30:175-180

8. Bech P, Austin SA, Lau M. Patient Reported Outcome Measures (PROMs): Examination of the psychometric properties of two measures for burden of symptoms and quality of life in patients with depression or anxiety. Nord J Psychiatry 2018;72:251-258.

9. Medical Research Council. Clinical trial of the treatment of depressive illness: report to the Medical Research Council by its Clinical Psychiatry Committee. Br Med J 1965;1: $881-886$

10. Johnstone EC, Deakin JF, Lawler P et al. The Northwick Park electroconvulsive therapy trial. Lancet 1980;2: 1317-1320.

11. Carney MW, Roth M, Garside RF. The Diagnosis of Depressive Syndromes and the prediction of E.C.T. response. Br J Psychiatry 1965;111: 659-674. 